\title{
The legend of plantar neuropraxia in long-distance athletes
}

\author{
D S Kellogg, ${ }^{1} \mathrm{MD} ; \mathrm{J}$ Joslin, ${ }^{2} \mathrm{MD}$ \\ ${ }^{1}$ Department of Emergency Medicine, Johns Hopkins University, Baltimore, Maryland, USA \\ ${ }^{2}$ Department of Emergency Medicine, State University of New York, Upstate Medical University, Syracuse, New York, USA
}

Corresponding author: J Joslin (joslinj@upstate.edu)

\begin{abstract}
Background. Legend has it that endurance athletes who develop plantar foot pain during long-distance running frequently experience an eventual relief of pain due to a transient neuropraxia brought on by continued activity.

Objective. To evaluate the nature of this legend, we assessed long-distance runners for the presence of sensory deficits before and after completion of an ultramarathon, expecting to find an induced neuropraxia and abnormal sensory results.

Methods. Twenty-five adult participants of an ultramarathon were evaluated prior to their $50 / 100 \mathrm{~km}$ run and again upon completion of the race. Neurosensory testing was performed using a $10 \mathrm{~g}$ monofilament at 4 locations on each foot and a $128 \mathrm{~Hz}$ tuning fork at one location on each foot. The same techniques were used prior to, and at conclusion of the race.

Results. We detected no neuropraxia or sensory deficits in any participant, despite reports by the same subjects that they had experienced the phenomenon during the race. While runners commonly report losing sensation in their feet during long runs, we were unable to demonstrate any sensory deficit with simple field-based testing.

Conclusion. We believe that there is room for additional research to be performed using more sensitive means of neurosensory evaluation.
\end{abstract}

S Afr J SM 2013;25(2):51-52. DOI:10.7196/SAJSM.468

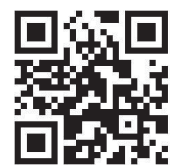

Legend has it, and anecdotal reports reinforce that endurance athletes who develop plantar foot pain during long-distance running events will frequently experience an eventual relief of pain due to a transient neuropraxia brought on by continued activity. Does this transient neuropraxia, which seems to occur during, or immediately after endurance running, represent an early symptom of the development of permanent neuropathy, or is it a benign and transient phenomenon? Previous research has considered permanent neurological deficits in runners, ranging from minor changes in sensory thresholds ${ }^{[1]}$ to entrapment neuropathies. ${ }^{[2,3]}$ A growing body of literature has investigated and reviewed these changes in pain perception among runners. ${ }^{[4-6]}$ Even though there is mention of acute neurosensory deficits during a race, in scholarly literature ${ }^{[1,2]}$ and among athletes, no studies have rigorously demonstrated it. Using a standardised field technique utilising monofilament testing, we sought to expand on previous research on neurosensory changes in athletes and discover if a detectable, objective neuropraxia was induced in ultramarathon runners on race day.

\section{Methods}

The study was reviewed and approved by the State University of New York Upstate Medical University Institutional Review Board. Runners participating in the 2010 Green Lakes Endurance Runs single-day $50 / 100 \mathrm{~km}$ trail races, were asked to participate in the study. Study participation was not a requirement for race participation, or to receive medical care during the race. All participants were aged $\geq 18$ years and provided informed, written consent prior to study participation. Participants were given a short questionnaire surveying demographic and running-related information.
Prior to the race, participants' feet were visually inspected for the presence of calluses and blisters. Two accepted methods for evaluating diabetic neuropathy ${ }^{[7]}$ were then used to evaluate vibratory and lighttouch sensation. First, a $128 \mathrm{~Hz}$ tuning fork was placed on the dorsum of the great toe; the test was considered positive if the participant could not feel the vibration of the tuning fork while the examiner could, and negative otherwise. Second, a $10 \mathrm{~g}$ monofilament was touched to the plantar aspect of the great toe, and the plantar aspects of the first, third, and fifth metatarsophalangeal joints; the test was considered positive if the participant was unable to feel the monofilament touching the foot when the examiner applied just enough pressure to cause the monofilament to bend.

The same evaluation of visual inspection, vibratory, and light-touch sensation was repeated on participants at either the completion of the race, or the time of discontinuation of the race. Results of both evaluations were matched by participant and compared for analysis.

\section{Results}

In total, 31 subjects ( 17 men, 14 women; age range 24 - 66 years) consented to study participation and completed the questionnaire; 30 participated in the initial neurosensory testing, and 26 in the postrace testing. One participant had post-race testing performed but not pre-race testing; therefore, only 25 participants were compared longitudinally. None had a diagnosed history of conditions that might have caused permanent neurosensory deficits. Interestingly, and germane to our investigation, 15 participants (48\%) reported having previously experienced neuropraxia associated with running.

None of the participants $(0 / 25 ; 0 \%)$ was found to have a difference in neurosensory test results when comparing pre- and post-race evaluation; none $(0 / 30 ; 0 \%)$ was found to have a deficit in vibration 
sensation prior to the race; and none $(0 / 26 ; 0 \%)$ was found to have impairment at re-evaluation post race. A few participants demonstrated deficits in light touch at one or more locations during pre-race evaluation $(4 / 30 ; 13 \%)$; however, they did not return for follow-up testing at the conclusion of the race.

The only significant, but not surprising difference between pre- and post-race evaluations was that more runners had blisters after the race $(13 / 26 ; 50 \%)$ than before the race $(2 / 30 ; 6.7 \%)$.

\section{Discussion}

We found no evidence of neuropraxia in our subjects. No prior reports have demonstrated transient plantar neuropraxia in endurance athletes. We therefore conclude that plantar neuropraxia among endurance athletes is either a somatoform manifestation, or was simply not detected by our evaluation. Almost half of our participants reported subjective neuropraxia at some point during their running careers. Indeed, some stated that they experienced this during the race on the day of the study. Despite these relevant experiences, none was objectively found to have a loss of sensation on post-race testing. Perhaps runners reporting these feelings are experiencing a somatoform phenomenon.

Other investigations evaluating pain perception among endurance athletes have shown that the fastest runners in an ultramarathon experienced a moderate reduction in pain perception immediately after a race. ${ }^{[5]}$ Runners may misinterpret decreased pain sensation as decreased overall sensation. It is also possible that athletes do develop a real sensory deficit, but that the act of sitting down and removing shoes to have testing performed may 'cure' the deficits.

Finally, it is possible that plantar neuropraxia among endurance athletes is real, but that our simple testing method was too insensitive to detect it. To allow for rapid field evaluation and encourage maximum study participation, we performed neurological testing methods typically used to assess diabetic neuropathy. Such techniques may not be sensitive enough to detect more subtle deficits encountered by runners. In the future, methods that may increase sensitivity for minor changes in perception include using a thinner monofilament $(1 \mathrm{~g})$ and additional modalities of sensory testing, to elucidate whether any neuropraxia that may exist affects nerve fibre types differentially.

Additionally, our study had a small sample size, and examined participants at only one event. Perhaps different race conditions
- e.g. running on the pavement v. dirt trails, changes in heat and humidity, etc., or a different athlete population - might have changed our findings. It is unfortunate that the only participants with pre-race deficits did not return for post-race testing, as their sensory changes may have been more pronounced.

If transient plantar neuropraxia is a real phenomenon, what is the likely mechanism? One mechanism proposed in the literature involves tight running shoes which may lead to ischaemia of the nerves to the foot. ${ }^{[2]}$ Prior studies have pointed to the repetitive trauma of running as a cause of plantar neuropathy. ${ }^{[3,8]}$ Medial plantar neuropathy (jogger's foot), neuropathic pain over the course of the medial plantar nerve, ${ }^{[3]}$ is a well-known phenomenon that occurs during and after exercise. Perhaps transient plantar neuropraxia is merely a minor form of, or a precursor to this condition. Or, maybe it is a side-effect of the betterstudied phenomenon of increased pain thresholds among athletes. ${ }^{[4-6]}$

If the phenomenon of plantar neuropraxia truly exists, and if its effects are not benign, we could potentially see more neurological sequelae as ultra-running, and other endurance sports requiring prolonged-time walking, hiking or running, become more popular. Further research is needed to explore what sort of damage transient and permanent athletes may be causing to the nerves in their feet and what mechanism may be the cause.

\section{References}

1. Dyck PJ, Classen SM, Stevens JC, O'Brien PC. Assessment of nerve damage in the feet of long-distance runners. Mayo Clin Proc 1987;62(7):568-572. [http://dx.doi. org/10.1016/S0025-6196(12)62294-0]

2. McKean KA. Neurologic running injuries. Neurol Clin 2008;26:281-296. [http:// dx.doi.org/10.1016/j.ncl.2007.11.007]

3. Peck E, Finnoff JT, Smith J. Neuropathies in runners. Clin Sports Med 2010;29:437547. [http://dx.doi.org/10.1016/j.csm.2010.03.002]

4. Droste C, Greenlee MW, Schreck M, Roskamm H. Experimental pain thresholds and plasma beta-endorphin levels during exercise. Med Sci Sports Exerc 1991;23(3):334342. [http://dx.doi.org/10.1249/00005768-199103000-00012]

5. Hoffman MD, Lee J, Zhao H, Tsodikov A. Pain perception after a running a 100mile ultramarathon. Arch Phys Med Rehabil 2007;88(8):1042-1048. [http://dx.doi. org/10.1016/j.apmr.2007.05.004]

6. Janal MA. Pain sensitivity, exercise and stoicism. J R Soc Med 1996;89:376-381.

7. Singh N, Armstrong DG, Lipsky BA. Preventing foot ulcers in patients with diabetes. JAMA 2005;293(2):217-228. [http://dx.doi.org/10.1001/jama.293.2.217]

8. Oh SJ, Meyer RD. Entrapment neuropathies of the tibial (posterior tibial) nerve. Neurol Clin 1999;17(3):593-615. [http://dx.doi.org/10.1016/S0733-8619(05)70154-7] 